Comment. Math. Helv. 75 (2000) 644-667

0010-2571/00/040644-24\$1.50+0.20/0
(C) 2000 Birkhäuser Verlag, Basel

Commentarii Mathematici Helvetici

\title{
Geometry for palindromic automorphism groups of free groups
}

Henry H. Glover and Craig A. Jensen

Dedicated to Peter Hilton:

Doc, Note, I Dissent. A FAST NEVER PREVEnts A FATNESS. I Diet ON COD.

\begin{abstract}
We examine the palindromic automorphism group $\Pi A\left(F_{n}\right)$ of a free group $F_{n}$, a group first defined by Collins in [5] which is related to hyperelliptic involutions of mapping class groups, congruence subgroups of $S L_{n}(\mathbb{Z})$, and symmetric automorphism groups of free groups. Cohomological properties of the group are explored by looking at a contractible space on which $\Pi A\left(F_{n}\right)$ acts properly with finite quotient. Our results answer some conjectures of Collins and provide a few striking results about the cohomology of $\Pi A\left(F_{n}\right)$, such as that its rational cohomology is zero at the vcd.
\end{abstract}

Mathematics Subject Classification (2000). 20F32, 20J05; 20F28, 55N91.

Keywords. Palindromes, hyperelliptic, free groups, moduli spaces, outer space, auter space.

\section{Introduction}

Let $\operatorname{Aut}\left(F_{n}\right)$ be the automorphism group of a free group $F_{n}$ on $n$ generators $a_{1}, a_{2}, \ldots, a_{n}$. A reduced word $x_{1}^{\epsilon_{1}} x_{2}^{\epsilon_{2}} \ldots x_{n}^{\epsilon_{n}}$ is called a palindrome if it is equal to its reverse $x_{n}^{\epsilon_{n}} x_{n-1}^{\epsilon_{n-1}} \ldots x_{1}^{\epsilon_{1}}$. In [2] Collins defines the palidromic automorphism group $\Pi A\left(F_{n}\right)$ as the subgroup of $A u t\left(F_{n}\right)$ consisting of all automorphisms $\alpha$ for which $\alpha\left(a_{i}\right)$ is a palindrome for all $i$. He showed that the group was generated by three types of automorphisms:

- Maps $\left(a_{i} \| a_{j}\right), i \neq j$, which send $a_{i} \mapsto a_{j} a_{i} a_{j}$ and fix all other generators $a_{k}$.

- Maps $\sigma_{a_{i}}$ which send $a_{i} \mapsto a_{i}^{-1}$ and fix all other generators $a_{k}$.

- Maps corresponding to elements of the symmetric group $\Sigma_{n}$ which permute the $a_{1}, \ldots, a_{n}$ among themselves.

The portion of $\Pi A\left(F_{n}\right)$ generated by just the $\left(a_{i} \| a_{j}\right)$ is called the elementary palindromic automorphism group of $F_{n}$ and denoted $E \Pi A\left(F_{n}\right)$. Note that $\Pi A\left(F_{n}\right)=$ $E \Pi A\left(F_{n}\right) \rtimes\left(\mathbb{Z} / 2 \succ \Sigma_{n}\right)$. Collins showed that a set of defining relators for $E \Pi A\left(F_{n}\right)$ is given by relations of the form

(1) $\left(a_{i} \| a_{k}\right)\left(a_{j} \| a_{k}\right)=\left(a_{j} \| a_{k}\right)\left(a_{i} \| a_{k}\right)$ 
(2) $\left(a_{i} \| a_{k}\right)\left(a_{j} \| a_{l}\right)=\left(a_{j} \| a_{l}\right)\left(a_{i} \| a_{k}\right)$

(3) $\left(a_{i} \| a_{k}\right)\left(a_{j} \| a_{k}\right)\left(a_{i} \| a_{j}\right)=\left(a_{i} \| a_{j}\right)\left(a_{j} \| a_{k}\right)\left(a_{i} \| a_{k}\right)^{-1}$

He remarked how similar this was to the relations for the pure symmetric automorphism group $P \sum A\left(F_{n}\right)$ (see Gilbert's work in [10]):

(1) $\left(a_{i} \mid a_{k}\right)\left(a_{j} \mid a_{k}\right)=\left(a_{j} \mid a_{k}\right)\left(a_{i} \mid a_{k}\right)$

(2) $\left(a_{i} \mid a_{k}\right)\left(a_{j} \mid a_{l}\right)=\left(a_{j} \mid a_{l}\right)\left(a_{i} \mid a_{k}\right)$

(3) $\left(a_{i} \mid a_{k}\right)\left(a_{j} \mid a_{k}\right)\left(a_{i} \mid a_{j}\right)=\left(a_{i} \mid a_{j}\right)\left(a_{j} \mid a_{k}\right)\left(a_{i} \mid a_{k}\right)$

where $\left(a_{i} \mid a_{j}\right), i \neq j$, sends $a_{i} \mapsto a_{j}^{-1} a_{i} a_{j}$ and fixes all other generators $a_{k}$.

On the basis of this, Collins conjectured that one could find the virtual cohomological dimension of $\Pi A\left(F_{n}\right)$ by employing the methods of [7], as he did for $\Sigma A\left(F_{n}\right)$ in [4]. He also speculated that $\operatorname{E\Pi A}\left(F_{n}\right)$ is torsion free, just as $P \Sigma A\left(F_{n}\right)$ is. We are able to answer both of these questions in this paper, as well as obtaining several interesting facts about the cohomology of $\Pi A\left(F_{n}\right)$.

Theorem 1.1. Let $\Pi A\left(F_{n}\right)$ be the palindromic automorphism group of the free group $F_{n}$ on $n$ letters and let $\operatorname{E\Pi A}\left(F_{n}\right)$ be the subgroup of elementary palindromic automorphisms. Then

a) The virtual cohomological dimension of $\Pi A\left(F_{n}\right)$ is $n-1$.

b) (i) For the prime 2, the Krull dimension of $\hat{H}^{*}\left(\Pi A\left(F_{n}\right) ; \mathbb{Z}_{(2)}\right)$ is $n$. For odd primes $p$, the Krull dimension of $\hat{H}^{*}\left(\Pi A\left(F_{n}\right) ; \mathbb{Z}_{(p)}\right)$ is $\left[\frac{n}{p}\right]$.

(ii) In the range where the Krull dimension of $\hat{H}^{*}\left(\Pi A\left(F_{n}\right) ; \mathbb{Z}_{(p)}\right)$ is 1 , the period is $2(p-1)$.

c) The group $E \Pi A\left(F_{n}\right)$ is torsion free.

d) The cohomology group $H^{n-1}\left(\Pi A\left(F_{n}\right) ; \mathbb{Q}\right)=0$.

e) If $p$ is an odd prime and $n=p, p+1, p+2$, then the Farrell cohomology of the palindromic automorphism group is the same as that of the symmetric group on $p$ elements:

$$
\hat{H}^{*}\left(\Pi A\left(F_{n}\right) ; \mathbb{Z}_{(p)}\right) \cong \hat{H}^{*}\left(\Sigma_{p} ; \mathbb{Z}_{(p)}\right) .
$$

For analogous results concerning $\operatorname{Aut}\left(F_{n}\right)$, see [3] and [11]. See [9] for the definition of the Farrell cohomology $\hat{H}^{*}(G ; M)$ of a group $G$ of finite vcd with coefficients in a $G$-module $M$ and also see [2] for several useful properties of these cohomology groups.

The remainder of this paper is structured as follows. In section 2, we discuss $\Pi A\left(F_{n}\right)$ and note how it relates to some other groups, while in section 3 we introduce the space $L_{\sigma_{n}}$ which $\Pi A\left(F_{n}\right)$ acts on and prove parts a) and d) of Theorem 1.1. Section 4 is concerned with a realization proposition which allows us to establish parts b) (i) and c) of the main theorem. Finally, section 5 looks in more detail at the cohomology of $\Pi A\left(F_{n}\right)$ at odd primes $p$ and establishes parts b) (ii) and e) of the main theorem.

The authors would like to thank John Meier for an enlightening conversation about symmetric automorphisms of free groups, aiding the presentation of this 
paper.

\section{Relationships with other groups}

Let $X_{n}$ be the spine of auter space (see [7], [13], [14]) and $Q_{n}=X_{n} / \operatorname{Aut}\left(F_{n}\right)$. Let $\sigma_{n} \in \operatorname{Aut}\left(F_{n}\right)$ be the automorphism which sends $a_{i} \mapsto a_{i}^{-1}$ for each $i$.

Define the $\theta$-graph $\theta_{m}$ to be a graph with 2 vertices and $m+1$ edges, where each edge goes from one vertex to the other one. Choose one of the two vertices of $\theta_{1}$ to be the basepoint $*$, and define the rose $R_{n}$ to be the result of wedging together $n$ copies of $\theta_{1}$ at the basepoint.

The petals of the rose $R_{n}$ can be identified with the generators $a_{i}$ of $F_{n}$, so that $\pi_{1}\left(R_{n}, *\right) \cong F_{n}$. There is an action of $\left\langle\sigma_{n}\right\rangle=\mathbb{Z} / 2$ on $R_{n}$ given by inverting each petal of the rose. This action realizes the subgroup $\left\langle\sigma_{n}\right\rangle$ in the sense of [22] (also cf. [6].) An action of a group $G$ on a graph $\Gamma$ is without inversions if $G$ does not send any edge $e$ to its inverse $\bar{e}$, and an action is reduced if there are no $G$-invariant subforests in $\Gamma$. The action of $\sigma_{n}$ on $R_{n}$ is both without inversions and reduced. From now on, when we refer to a group action on a graph, it is assumed that the edges of the graph are subdivided as necessary to insure that the group acts without inversions.

Note that the palindromic automorphism group $\Pi A\left(F_{n}\right)$ is just $C_{A u t\left(F_{n}\right)}\left(\sigma_{n}\right)$. This follows because an easy argument shows that every element of $C_{A u t\left(F_{n}\right)}\left(\sigma_{n}\right)$ is palindromic, and because the generators of $\Pi A\left(F_{n}\right)$ are all in $C_{A u t\left(F_{n}\right)}\left(\sigma_{n}\right)$. For example, the generators $\left(a_{i} \| a_{j}\right)$ are just products of $\sigma_{n}$-Nielsen transformations (see [20] where the $G$-Nielsen transformation $\langle e, f\rangle \Gamma$ of a $G$-graph $\Gamma$ has the same vertex and edge set as $\Gamma$ but where the terminal point of an edge $e g, g \in G$, in the new graph is the initial point of the edge $f g$ in the original graph; this induces a map $\langle e, f\rangle$ from the fundamental groupoid of the first graph to that of the second where $e g$ is sent to $(e f) g$ and all other edges are sent to themselves.) That is, if the petal $a_{i}$ of the rose consists of the edges $\bar{e}_{i} f_{i}$, then $\left(a_{i} \| a_{j}\right)$ is the composition $\left\langle e_{i}, \bar{f}_{j}\right\rangle \circ\left\langle e_{i}, e_{j}\right\rangle$.

As a note for the curious, it follows that $\Pi A\left(F_{n}\right)$ and $\Sigma A\left(F_{n}\right)$ are distinct groups for $n \geq 2$, since a direct argument shows that $\Sigma A\left(F_{n}\right)$ has no element of order 2 in its center. In addition, $E \Pi A\left(F_{n}\right)$ and $P \Sigma A\left(F_{n}\right)$ are also obviously distinct (for $n \geq 3$; for $n=1,2$ they are the same group with the same presentation,) since the former abelianizes to an elementary abelian 2-group of rank $n(n-1)$ while the latter abelianizes to a free abelian group of rank $n(n-1)$.

In addition to its formal palindromic properties, the group $\Pi A\left(F_{n}\right)$ arises naturally from looking at hyperelliptic subgroups of mapping class groups (cf. Gries [12] for corresponding homological properties.) We have a commutative diagram

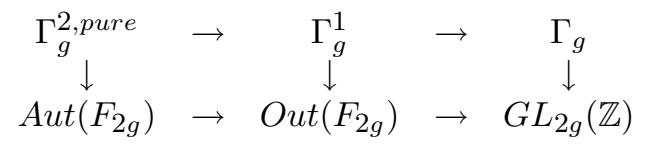


where $\Gamma_{g}$ is the mapping class group of an orientable surface of genus $g, \Gamma_{g}^{1}$ is the mapping class group of an orientable surface of genus $g$ with 1 puncture, and $\Gamma_{g}^{2, \text { pure }}$ is the mapping class group of an orientable surface of genus $g$ with two punctures, where each puncture is fixed pointwise. The map from $\Gamma_{g}^{2, \text { pure }}$ to $\operatorname{Aut}\left(F_{2 g}\right)$ is obtained first by taking an intersection basis $a_{1}, b_{1}, \ldots, a_{g}, b_{g}$ for the fundamental group of the surface $S$ with two punctures. One of the punctures should serve as the basepoint for fundamental group considerations. The other is treated as an actual puncture, so that the fundamental group of the surface minus this point is a free group $F_{2 g}$ on $2 g$ generators. The map from $\Gamma_{g}^{2, \text { pure }}$ to $\operatorname{Aut}\left(F_{2 g}\right)$ is now obtained by sending an element of $\Gamma_{g}^{2, \text { pure }}$ to the automorphism of $F_{2 g}$ that it induces. The map from $\Gamma_{g}^{1}$ to $\operatorname{Out}\left(F_{2 g}\right)$ is obtained similarly.

Let $\psi \in \Gamma_{g}^{2, \text { pure }}$ be a hyperelliptic involution (see, for example, [8].) Then $\psi$ has $2 g+2$ fixed points, two of which are of course the punctures on the surface $S$. Choose loops $a_{1}, b_{1}, \ldots, a_{g}, b_{g}$, based at one of the punctures, which form an intersection basis for the surface $S$ (say the ones described in [8] in the section on hyperelliptic Riemann surfaces.) By going along the top row of diagram 2.1 and then projecting downward, we see that the image of $\psi$ in $G L_{2 g}(\mathbb{Z})$ is $-I$. Let $\bar{\psi}$ be the image of $\psi$ in $\operatorname{Aut}\left(F_{2 g}\right)$. Our goal is to show that $\bar{\psi}$ is conjugate to $\sigma_{2 g}$ in $\operatorname{Aut}\left(F_{2 g}\right)$.

Lemma 2.2. If $\phi \in \operatorname{Aut}\left(F_{n}\right)$ and the image of $\phi$ in $G L_{n}(\mathbb{Z})$ is $-I$, then the image of any conjugate $\alpha^{-1} \phi \alpha$ of $\phi, \alpha \in \operatorname{Aut}\left(F_{n}\right)$, is also $-I$.

Proof. This follows directly since $-I$ is in the center of $G L_{n}(\mathbb{Z})$.

Lemma 2.3. If $\phi \in \operatorname{Aut}\left(F_{n}\right)$ is an involution whose image in $G L_{n}(\mathbb{Z})$ is $-I$, then $\phi$ can be realized on a marked graph whose underlying graph is the rose.

Proof. Realize $\phi$ on a reduced marked graph whose underlying graph is $\Lambda$. First, we show that if $e$ is an edge of $\Lambda$ which is not fixed by $\phi$, then we can assume that one endpoint of $e$ is the basepoint $*$. Let $f=\phi(e)$. Choose a shortest path $\gamma$ from $e$ to $*$. Since $\operatorname{stab}(e)=\langle 1\rangle, \operatorname{stab}(e) \subseteq \operatorname{stab}(h)$ for every $h$ in the path $\gamma$. So we can apply a sequence of Nielsen transformations (see [20]) and slide $e$ along $\gamma$ to *. Note that since $\Lambda$ is reduced, $\{e, f\}$ now forms either a rose $R_{2}$ based at $*$, or a $\theta_{1}$. Proceeding in this manner, we can slide all of the edges of $\Lambda$ not fixed by $\phi$ to the basepoint.

By way of contradiction, suppose that two of these $\theta_{1}$-graphs (that have been moved so that one vertex of each $\theta_{1}$ is the basepoint), say $\left\{e_{1}, f_{1}\right\}$ and $\left\{e_{2}, f_{2}\right\}$, share another common vertex in addition to the basepoint $*$. That is, suppose that there is a vertex $v \neq *$ and each of $e_{1}, f_{1}, e_{2}, f_{2}$ go from $v$ to $*$. Say $g: R_{n} \rightarrow \Lambda$ is the marked graph, and recall that $\pi_{1}\left(R_{n}\right)=\left\langle a_{1}, \ldots, a_{n}\right\rangle=F_{n}$. By replacing $\phi$ by a conjugate if necessary (see Lemma 2.2) we can assume that $g$ sends the petal $a_{1}$ of $R_{n}$ to $e_{1}^{-1} f_{1}$, the petal $a_{2}$ to $e_{2}^{-1} f_{2}$, and the petal $a_{3}$ of $R_{n}$ to $e_{1}^{-1} f_{2}$. So in 
$\pi_{1}(\Lambda)$ we have

$$
\begin{aligned}
& \phi \cdot a_{1}=f_{1}^{-1} e_{1}=g\left(a_{1}^{-1}\right), \\
& \phi \cdot a_{2}=f_{2}^{-1} e_{2}=g\left(a_{2}^{-1}\right),
\end{aligned}
$$

and

$$
\phi \cdot a_{3}=f_{1}^{-1} e_{2}=g\left(a_{1}^{-1} a_{3} a_{2}^{-1}\right) .
$$

Hence the first column of $i m(\phi)$ in $G L_{n}(\mathbb{Z})$ is $(-1,0, \ldots, 0)$, the second column is $(0,-1,0, \ldots, 0)$, and the third column is $(-1,-1,1,0, \ldots, 0)$. This contradicts the fact that $i m(\phi)=-I$.

If the result of sliding to the basepoint $*$ all edges of $\Lambda$ not fixed by $\phi$ yields a rose $R_{n}$, then we are done. Otherwise, suppose by way of contradiction that there exist edges $e, f$, and $h$ of $\Lambda$ such that

- Both $e$ and $f$ go from some vertex $v \neq *$ to $*$.

- $\phi(e)=f$.

- $h$ goes from $v$ to $v$.

- $\phi(h)=h$.

As before, say $g: R_{n} \rightarrow \Lambda$ is the marked graph. By replacing $\phi$ by a conjugate if necessary (see Lemma 2.2) we can assume that $g$ sends the petal $a_{1}$ of $R_{n}$ to $e^{-1} f$ and the petal $a_{2}$ of $R_{n}$ to $e^{-1} h f$. So in $\pi_{1}(\Lambda)$ we have

$$
\phi \cdot a_{1}=f^{-1} e=g\left(a_{1}^{-1}\right)
$$

and

$$
\phi \cdot a_{2}=f^{-1} h e=\left(e^{-1} f\right)^{-1}\left(e^{-1} h f\right)\left(e^{-1} f\right)^{-1}=g\left(a_{1}^{-1} a_{2} a_{1}^{-1}\right) .
$$

Hence the first column of $i m(\phi)$ in $G L_{n}(\mathbb{Z})$ is $(-1,0, \ldots, 0)$ and the second column is $(-2,1,0, \ldots, 0)$. This contradicts the fact that $i m(\phi)=-I$.

Proposition 2.4. If $\phi \in \operatorname{Aut}\left(F_{n}\right)$ is an involution whose image in $G L_{n}(\mathbb{Z})$ is $-I$, then $\phi$ is conjugate in $\operatorname{Aut}\left(F_{n}\right)$ to $\sigma_{n}$.

Proof. Realize $\phi$ on a marked graph $g: R_{n} \rightarrow R_{n}$. By replacing $\phi$ by a conjugate if necessary, we can assume $g\left(a_{i}\right)=a_{i}$ for all $i$. The involution $\phi$ of the graph $R_{n}$ must send the petal $a_{1}$ to some $a_{j}^{ \pm 1}$, since it is a graph automorphism. But since $i m(\phi)=-I \in G L_{n}(\mathbb{Z})$, we see that $a_{j}^{ \pm 1}$ must be $a_{1}^{-1}$. Similarly, we can see that the graph automorphism $\phi$ sends, for each $i$, the petal $a_{i}$ to the petal $a_{i}^{-1}$. This means our current $\phi$ is equal to $\sigma_{n}$ (and thus our original $\phi$, before we replaced it by a conjugate, was conjugate to $\sigma_{n}$.)

The following corollary is immediate:

Corollary 2.5. The image $\bar{\psi}$ of $\psi$ in Aut $\left(F_{2 g}\right)$ is conjugate to $\sigma_{2 g}$. Hence the hyperelliptic subgroup $C_{\Gamma_{g}^{2, \text { pure }}}(\psi)$ is conjugate to a subgroup of $C_{A u t\left(F_{2 g}\right)}\left(\sigma_{2 g}\right)$. 
As remarked in [5], the image of $\Pi A\left(F_{n}\right)$ in $G L_{n}(\mathbb{Z})$ is the subgroup of $G L_{n}(\mathbb{Z})$ consisting of invertible matrices where each column has exactly one odd entry (and the rest are even.) The subgroup is the semidirect product $\tilde{\Gamma}_{2}(\mathbb{Z}) \rtimes \Sigma_{n}$ where $\tilde{\Gamma}_{2}(\mathbb{Z})$ is the 2 -congruence subgroup defined by the short exact sequence

$$
\langle 1\rangle \rightarrow \tilde{\Gamma}_{2}(\mathbb{Z}) \longmapsto G L_{n}(\mathbb{Z}) \rightarrow G L_{n}(\mathbb{Z} / 2) \rightarrow\langle 1\rangle
$$

and $\Sigma_{n}$ is standard inclusion of the symmetric group

$$
\Sigma_{n} \subset G L_{n}(\mathbb{Z}) .
$$

\section{A space for $\Pi A\left(F_{n}\right)$ to act on}

We define a certain contractible space $L_{\sigma_{n}}$, related to auter space, which $\Pi A\left(F_{n}\right)$ acts on with finite stabilizers and finite quotient. This allows us to obtain some cohomological results.

A graph $\Gamma$ is a $\theta_{1}$-tree of rank $n$ if there exists a pointed tree $T$ such that $\Gamma$ is obtained by "doubling" every edge of $T$ into a $\theta_{1}$-graph. That is, the vertex set of $\Gamma$ is the same as the vertex set of $T$ and for every edge $e$ of $T$ going from $v$ to $w, \Gamma$ has two edges $e_{1}$ and $e_{2}$, both of which go from $v$ to $w$. There is a natural $\mathbb{Z} / 2$-action on such a graph $\Gamma$, which is given by switching the two edges in each $\theta_{1}$-graph. Note that the orbit space of $\Gamma$ under this action is just the tree $T$.

Claim 3.1. The reduced graphs $\Gamma$ which realize the subgroup $\left\langle\sigma_{n}\right\rangle$ of $\operatorname{Aut}\left(F_{n}\right)$ are exactly the $\theta_{1}$-trees of rank $n$, where $\sigma_{n}$ acts on the trees via their natural $\mathbb{Z} / 2$-action.

Proof. We have already mentioned that the rose $R_{n}$ realizes $\sigma_{n}$. From Theorem 2 of [20], the other reduced graphs $\Gamma$ which also realize $\sigma$ are those that are Nielsen equivalent to $R_{n}$ (up to an equivariant isomorphism.)

If $e$ is an edge in one of the copies of $\theta_{1}$ in $R_{n}$ and $f$ is an edge in a different $\theta_{1}$ in $R_{n}$, and both $e$ and $f$ point toward the basepoint, then Nielsen transformation $\langle e, f\rangle$ has the result of pulling the $\theta_{1}$-graph $\left\{e, \sigma_{n} e\right\}$ through the $\theta_{1}$-graph $\left\{f, \sigma_{n} f\right\}$, so that now $e$ terminates at the initial vertex of $f$, rather than at the basepoint $*$. In other words, the result of applying one Nielsen transformation to $R_{n}$ is that of sliding one of the petals of $R_{n}$ up though another petal.

A basic induction argument now yields that the result of applying a series of Nielsen transformations fo $R_{n}$ will be some $\theta_{1}$-tree $\Gamma$.

Recall from [21] that an edge $e$ of a $G$-graph $\Gamma$ is inessential if it is in every maximal $G$-invariant subforest of $\Gamma$. A $G$-graph $\Gamma$ is inessential if it has at least one inessential edge and is essential if it is not inessential. Let $X_{n}^{G}$ be the fixed 
point subspace of $X_{n}$ corresponding to some finite subgroup $G$ of $\operatorname{Aut}\left(F_{n}\right)$. From [17] (cf. part III of [18] and [21]), both the centralizer $C_{A u t\left(F_{n}\right)}(G)$ and the normalizer $N_{A u t\left(F_{n}\right)}(G)$ act on the contractible space $X_{n}^{G}$ with finite stabilizers and finite quotient. Moreover, the space $X_{n}^{G} G$-equivariantly deformation retracts to the space $L_{G}$, where $L_{G}$ is constructed from $X_{n}^{G}$ by considering only essential marked graphs. Hence $L_{G}$ is a good space to study if one wishes to calculate the cohomology of $C_{\text {Aut }\left(F_{n}\right)}(G)$ or $N_{\text {Aut }\left(F_{n}\right)}(G)$.

Further recall that a $G$-graph $\hat{\Gamma}$ is a $G$-equivariant blowup of a $G$-graph $\Gamma$ if some $G$-invariant subforest $F$ of $\hat{\Gamma}$ can be collapsed away to yield $\Gamma$. Let $\phi: R_{n} \rightarrow \Gamma$ be some reduced marked graph realizing $\left\langle\sigma_{n}\right\rangle$. From Claim 3.1, $\Gamma$ is a $\theta_{1}$-tree. Blow up $\Gamma \sigma_{n}$-equivariantly to some maximal essential blowup $\hat{\Gamma}$.

Claim 3.2. The fixed points/cells of the action of $\sigma_{n}$ on $\hat{\Gamma}$ are exactly the valence 2 vertices of $\hat{\Gamma}$.

Proof. Note that vertices in $\hat{\Gamma}$ have valence 2 or 3 and that $\hat{\Gamma}$ is obtained from $\Gamma$ by blowing up an oriented ideal forest (see [17], [21], [18].) Briefly, ideal edges (oriented ideal forests) correspond to subsets of edges (chains of subsets of edges) which are pulled away from existing vertices in order to create new graphs which collapse down to the original graph.)

No edge is in $F i x_{\sigma_{n}}(\hat{\Gamma})$ because no edge is in $F i x_{\sigma_{n}}(\Gamma)$ and so blowing up ideal edges will not create any new edges that are fixed under the action of $\sigma_{n}$ (see [21] page 229.)

If a valence 3 vertex is in Fix $_{\sigma_{n}}(\hat{\Gamma})$, then at least one edge of $\hat{\Gamma}$ must be fixed by $\sigma_{n}$, which is a contradiction.

All of the valence 2 vertices of $\Gamma$ are in $F_{i x}(\Gamma)$. New valence 2 vertices created as ideal edges are blown up correspond to either:

- Old vertices of $\Gamma$ that used to be valence higher than 2 but have since had edges stripped (pulled away) from them. These are in $F i x_{\sigma_{n}}(\hat{\Gamma})$ because all vertices of $\Gamma$ are in $F i x_{\sigma_{n}}(\Gamma)$.

- New valence 2 vertices inserted to insure that $\sigma_{n}$ acts on $\hat{\Gamma}$ without inversions. These are also clearly in $F i x_{\sigma_{n}}(\hat{\Gamma})$.

Note that $* \in F i x_{\sigma_{n}}(\Gamma)$. Cut $\hat{\Gamma}$ along each of its valence 2 vertices, yielding a graph $\hat{\Gamma}_{\text {cut }}$ with the same number of valence 3 vertices and edges as $\hat{\Gamma}$ had, no valence 2 vertices, and twice as many valence 1 vertices as $\hat{\Gamma}$ had valence 2 vertices.

\section{Claim 3.3.}

$$
\hat{\Gamma}_{c u t}=\hat{\Gamma}_{1} \amalg \hat{\Gamma}_{1},
$$

the disjoint union of two trees $\hat{\Gamma}_{1}$ and $\hat{\Gamma}_{2}$, where $\hat{\Gamma}_{2}=\sigma_{n} \hat{\Gamma}_{1}$.

Proof. There is a covering map $p: \hat{\Gamma}_{c u t} \rightarrow \hat{\Gamma} / \sigma_{n}$ obtained by mapping to the orbit 
space under the $\sigma_{n}$-action. The forest collapse that sends $\hat{\Gamma}$ to $\Gamma$ is $\sigma_{n}$-equivariant, so it descends to a forest collapse of $\hat{\Gamma} / \sigma_{n}$ to $\Gamma / \sigma_{n}$. (That the quotient of the forest upstairs in $\hat{\Gamma}$ is also a forest in $\hat{\Gamma} / \sigma_{n}$ can be seen by an easy Euler characteristic argument.) But $\Gamma$ is a $\theta_{1}$-tree with a known $\sigma_{n}$-action on it, and $\Gamma / \sigma_{n}$ is a tree (in fact, the underlying tree of the $\theta_{1}$-tree.) Hence $\hat{\Gamma} / \sigma_{n}$ is a tree. Since $p: \hat{\Gamma}_{c u t} \rightarrow \hat{\Gamma} / \sigma_{n}$ is a covering map with fiber two points, $\hat{\Gamma}_{c u t}$ is as described.

Let $T$ be a pointed tree with $2 n-1$ edges, all vertices valence either 1 or 3 , where $*$ is one of the valence 1 vertices. (Then $T$ has $n+1$ valence 1 vertices and $n-1$ valence 3 vertices.) Let $T_{1}$ and $T_{2}$ be two isomorphic copies of $T$, and let $f: T_{1} \rightarrow T_{2}$ be an isomorphism. Define

$$
\Gamma_{T}=\frac{T_{1} \amalg T_{2}}{f(v) \sim v, \text { for all valence } 1 \text { vertices } v \text { of } T_{1}} .
$$

Define a $\sigma_{n}$-action on $\Gamma_{T}$ by

$$
\sigma_{n} x= \begin{cases}f(x), & x \in T_{1} \\ f^{-1}(x), & x \in T_{2}\end{cases}
$$

Proposition 3.4. There is a bijective correspondence between trees $T$ as above (with $2 n-1$ edges, etc) and maximal, essential blowups $\hat{\Gamma}$ of reduced $\sigma_{n}$-graphs. The bijection is given by $T \mapsto \Gamma_{T}$.

Proof. From claims 3.2 and 3.3, all blowups $\hat{\Gamma}$ have the required form. Finally, any $\Gamma_{T}$ can easily be reduced to a $\theta_{1}$-tree by collapsing edges, meaning that it is the blowup of such a graph.

All maximal simplices in $L_{\sigma_{n}}$ have the same dimension, from [21]. Maximal simplices in $L_{\sigma_{n}} / \Pi A\left(F_{n}\right)$ are constructed by taking chains of forest collapses from maximal blowups $\Gamma_{T}$. Alternatively, we can define a subforest of $T$ to be a collection $S$ of edges of $T$ such that there is no path in $S$ from one valence 1 vertex to another. (If there were such a path, then $S \cup \sigma_{n} S$ would be a cycle in $\Gamma_{T}$.) In this way, we can think of maximal simplices as coming from chains of subforests of various trees $T$.

Proof of part a) of Theorem 1.1: Since $\Pi A\left(F_{n}\right)$ acts on the contractible space $L_{\sigma_{n}}$ with finite stabilizers and finite quotient, the vcd of $\Pi A\left(F_{n}\right)$ is at most the dimension of a maximal cell from $L_{\sigma_{n}} / \Pi A\left(F_{n}\right)$. Such a cell comes from a chain of forest collapses of a tree $T$ with $2 n-1$ edges, $n+1$ valence 1 vertices, and $n-1$ valence 3 vertices. Hence we can collapse at most $n-1$ of the valence 3 vertices into other vertices while doing forest collapses, resulting in maximal simplices of dimension $n-1$. 
To show that the vcd of $\Pi A\left(F_{n}\right)$ is at least $n-1$, we note that the subgroup generated by $\left(a_{i} \| a_{n}\right)$ for $i \in\{1,2, \ldots, n-1\}$ is isomorphic to $\mathbb{Z}^{n-1}=\mathbb{Z} \times \ldots \times \mathbb{Z}$.

Lemma 3.5. Let $\tilde{\Gamma}$ be the underlying graph of a particular marked graph in $L_{\sigma_{n}}$. Hence $\tilde{\Gamma}$ comes equipped with a $\sigma_{n}$-action. Let $C$ be a simple closed curve in $\tilde{\Gamma}$. Then Fix $x_{\sigma_{n}}(C)$ contains exactly two points, and $\sigma_{n}(C)$ is the curve $-C$, or $C$ with the opposite of its original orientation.

Proof. $\tilde{\Gamma}$ can be blown up (not necessarily uniquely) to some maximal essential graph $\Gamma=\Gamma_{T}$. $\Gamma$ is the union of two isomorphic copies $T_{1}$ and $T_{2}$ of $T$, where $T_{1}$ and $T_{2}$ are attached along their corresponding valence 1 vertices.

As we collapse from $\Gamma$ to $\tilde{\Gamma}$, the trees $T_{1}$ and $T_{2}$ collapse to trees $\tilde{T}_{1}$ and $\tilde{T}_{2}$. However, the attaching points for $\tilde{T}_{1}$ and $\tilde{T}_{2}$ are no longer necessarily just the valence 1 vertices, and could be other vertices as well.

Let $\alpha_{1}$ be a taut path in $\tilde{T}_{1}$ from one attaching point $v_{1}$ to some other attaching point $v_{2}$, where furthermore there are no attaching points in the interior of $\alpha_{1}$. Let $\alpha_{2}=\sigma_{n}\left(\alpha_{1}\right)$ be the corresponding path in $\tilde{T}_{2}$. Then $\alpha_{1} \bar{\alpha}_{2}$ is a simple closed curve and $\sigma_{n}\left(\alpha_{1} \bar{\alpha}_{2}\right)=\alpha_{2} \bar{\alpha}_{1}$, or the original curve oriented in the other direction. Hence the curve $\alpha_{1} \bar{\alpha}_{2}$ satisfies the conclusions of the lemma. Our goal is to show that any simple closed curve $C$ takes this form.

Let $C_{1}$ be the portion of $C$ that is in $\tilde{T}_{1}$ and let $C_{2}$ be the portion of $C$ that is in $\tilde{T}_{2}$. Since $C$ is a cycle and yet both $\tilde{T}_{1}$ and $\tilde{T}_{2}$ are trees, both $C_{1}$ and $C_{2}$ are nonempty. In fact, there must be a path $\alpha_{1}$ in $C_{1}$ from one attaching point $v_{1}$ to some other attaching point $v_{2}$, where there are no other attaching points in the interior of the path. Since $C$ is a simple closed curve, $\alpha_{1}$ must be a taut path. Let $\alpha_{2}=C-\alpha_{1}$, some other taut path in $\tilde{\Gamma}$ from $v_{1}$ to $v_{2}$.

Let $p: \tilde{\Gamma} \rightarrow \tilde{T}_{1}$ be the map given by taking the quotient space under the action of $\sigma_{n}$. Note that $p\left(\alpha_{2}\right)$ is a path from $v_{1}$ to $v_{2}$. Since $\tilde{T}_{1}$ is a tree and $\alpha_{1}$ is the unique taut path in $\tilde{T}_{1}$ from $v_{1}$ to $v_{2}$, this gives us that edges $\left(\alpha_{1}\right) \subseteq \operatorname{edges}\left(p\left(\alpha_{2}\right)\right)$. Hence if $e$ is an edge in $\alpha_{1}$, then $p^{-1}(e)$ is two edges, $e \in C_{1}$ and $\sigma_{n}(e) \in C_{2}$. It follows that all of the oriented edges of the simple closed curve $\alpha_{1} \sigma_{n}\left(\bar{\alpha}_{1}\right)$ are in the simple closed curve $C$. Hence $C=\alpha_{1} \sigma_{n}\left(\bar{\alpha}_{1}\right)$.

Lemma 3.6. Let $\tilde{\Gamma}$ be the underlying graph of a particular marked graph in $L_{\sigma_{n}}$. Hence $\tilde{\Gamma}$ comes equipped with a $\sigma_{n}$-action. Choose an edge e in $\tilde{\Gamma}$. Among all simple closed curves $D$ which pass through e, choose one curve $C$ for which the distance from the curve to the basepoint $*$ is minimal. Then Fix $\sigma_{n}(C)$ is two points $v_{1}$ and $v_{2}$. One of these two points in the closest point in $C$ to the basepoint * and one of them is the farthest point in $C$ to the basepoint *.

Proof. Using the notation of the proof of Lemma 3.5, $C$ is the result of following some path $\alpha_{1}$ in $\tilde{T}_{1}$ and then $\bar{\alpha}_{2}=\sigma_{n}\left(\bar{\alpha}_{1}\right)$ in $\tilde{T}_{2}$, where $\alpha_{1}$ goes from the attaching 
point $v_{1}$ to the attaching point $v_{2}$.

Since $\alpha_{1}$ is a path in a pointed tree, there is a unique vertex $w$ in $\alpha_{1}$ which is closest to $*$. By way of contradiction, suppose that $w \notin\left\{v_{1}, v_{2}\right\}$. Then $w$ is not an attaching point. Let $\beta_{1}$ be the unique taut path in $\tilde{T}_{1}$ from $*$ to $w$. Let $\gamma_{1}$ be the unique subpath of $\beta_{1}$ which contains $w$ and exactly one attaching point $y$. Now let $\delta_{1}$ be the path in $\tilde{T}_{1}$ which starts at $y$, follows $\gamma_{1}$ along to $w$, and then either follows $C$ from $w$ to $v_{2}$ or $-C$ from $w$ to $v_{1}$ (where we choose whichever possibility insures that $\pm e \in \delta_{1}$.) Then the simple closed curve $\delta_{1} \sigma_{n}\left(\bar{\delta}_{1}\right)$ is closer to $*$ than $C$ is, which is a contradiction. Hence $w$ is $v_{1}$ or $v_{2}$, and the lemma follows.

Proposition 3.7. Let $\tilde{\Gamma}$ be a graph which occurs as an underlying graph of marked graphs in $L_{\sigma_{n}}$. Then there is only one possible $\sigma_{n}$-action on $\tilde{\Gamma}$.

Proof. We see that our task is to show that a unique $\sigma_{n}$-action is determined by the properties about simple closed curves listed in Lemmas 3.5 and 3.6.

Define an action $\eta$ on $\tilde{\Gamma}$ as follows. Let $e$ be an oriented edge of $\tilde{\Gamma}$. Among all simple closed curves $D$ which pass through $e$, choose one path $C$ for which the distance from the curve to the basepoint $*$ is minimal. Let $v_{1}$ be a point on $C$ which is closest to the basepoint. Let $n$ be the edge-path distance in $C$ from $v_{1}$ to $e$. Then there is an orientation $\epsilon \in\{-1,1\}$ such that if you traverse $\epsilon C$ starting at $v_{1}$ and go $n$ edges, you get to $e$. Define $\eta(e)$ to be the result of traversing $-\epsilon C$ starting at $v_{1}$ and then going $n$ more edges.

By Lemma 3.5 and 3.6, the action $\eta$ is well defined and if any $\sigma_{n}$ acts on $\tilde{\Gamma}$, then the $\sigma_{n}$-action and the $\eta$-action coincide.

Denote by $Q_{\sigma_{n}}$ the quotient space $L_{\sigma_{n}} / \Pi A\left(F_{n}\right)$.

Corollary 3.8. If two marked graphs in $L_{\sigma_{n}}$ have the same underlying graph, then they correspond to the same vertex in $Q_{\sigma_{n}}$. That is, the moduli space $Q_{\sigma_{n}}$ can be formed by looking only at the poset structure of the underlying graphs of marked graphs in $L_{\sigma_{n}}$.

Proof. From Proposition 3.7, any underlying graph of a marked graph in $L_{\sigma_{n}}$ has only one possible $\sigma_{n}$-action. But from Corollary 10.4 of [21], $\Pi A\left(F_{n}\right)$ acts transitively on the set of marked $\sigma_{n}$-graphs based on the same $\sigma_{n}$-graph. The result follows.

The simplices of $L_{\sigma_{n}}$ group themselves into cubes, as described in $\S 3$ of [15]. In [13], Hatcher and Vogtmann show that the quotients in $Q_{n}$ of cubes in $X_{n}$ have the rational homology of balls. They use this to create a cubical chain complex which has the same rational homology as $Q_{n}$. Our goal here is to establish a similar result for the cubes of maximal dimension in $Q_{\sigma_{n}}$, where this time we want the quotients of cubes to have the $\mathbb{Z}_{(p)}$-cohomology of balls, where $p$ is any odd prime. 
Following [14], we consider a maximal cube in $L_{\sigma_{n}}$. It is given by considering a maximal essential marked graph $\phi: R_{n} \rightarrow \Gamma_{T}$ and considering some maximal subforest $S$ of $T$. Recall that by a subforest of $T$ we mean a subset $S$ of the edges of $T$ where there is no path in $S$ from one valence 1 vertex of $T$ (or equivalently, one terminal edge of $T$ ) to another. From part a) of Theorem 1.1, $S$ has $n-1$ edges in it. The cube corresponding to the pair $(T, S)$ can thus be thought of as imbedded in $\mathbb{R}^{n-1}$, where each coordinate vector is an edge of the cube, the graph obtained by collapsing each edge of $S \cup \sigma_{n}(S)$ is at the origin, and $\Gamma_{T}$ is at $(1,1, \ldots, 1)$. Let $\operatorname{Aut}(T, S)$ be the group of all (pointed) automorphisms of the tree $T$ which take $S$ to $S$. The group $\operatorname{stab}_{\Pi A\left(F_{n}\right)}(T, S)=\left\langle\sigma_{n}\right\rangle \times \operatorname{Aut}(T, S)$ acts linearly on the cube by permuting the coordinates of $\mathbb{R}^{n-1}$, and fixes the diagonal from $(0,0, \ldots, 0)$ to $(1,1, \ldots, 1)$. (The involution $\sigma_{n}$ acts trivially on the cube, of course, since all of these cubes are coming from $L_{\sigma_{n}} \subset X_{n}^{\sigma_{n}}$.) Hence, just as in [15], the quotient of the cube in $Q_{\sigma_{n}}$ is a cone with base $S^{n-2} / A u t(T, S)$, where $S^{n-2}$ is the boundary of the cube.

Lemma 3.9. The finite group Aut(T) (and hence its subgroup $\operatorname{Aut}(T, S)$ ) is all 2-torsion.

Proof. Let $\xi \in A u t(T)$. Now $\xi$ must take the basepoint to the basepoint, and so it must take the unique edge attached to the basepoint to itself. For each $n$, let $E_{n}$ be the edges in $T$ which are at most distance $n$ from $*$. So $E_{0}$ is just one edge, and $\xi$ fixes it as already mentioned. Since all nonterminal vertices of $T$ have valence 3 , an inductive argument yields that $\xi^{2^{n}}$ fixes $E_{n}$ pointwise.

The following is an analog of Proposition 3.1 of [15]:

Proposition 3.10. $S^{n-2} / \operatorname{Aut}(T, S)$ has the $\mathbb{Z}_{(p)}$-cohomology of an $(n-2)$-sphere or a ball. The latter possibility happens when there is an element of Aut $(T, S)$ which induces an odd permutation of the edges of $S$.

Proof. The finite group $\operatorname{Aut}(T, S)$, which is all 2-torsion, acts cellularly on $S^{n-2}$, where the stabilizer of a cell fixes it pointwise. We use the spectral sequence for equivariant cohomology (cf. [2] VII $\S 7$ ):

$$
E_{1}^{r, s}=\prod_{[\delta] \in \Delta_{n}^{r}} H^{s}\left(\operatorname{stab}(\delta) ; \mathbb{Z}_{(p)}\right) \Rightarrow H_{\operatorname{Aut}(T, S)}^{r+s}\left(S^{n-2} ; \mathbb{Z}_{(p)}\right)
$$

where $[\delta]$ ranges over the set $\Delta^{r}$ of orbits of $r$-simplices $\delta$ in $S^{n-2}$. Since $A u t(T, S)$ is all 2-torsion and finite, so are all of the $\operatorname{stab}(\delta)$. Hence if $s>0, H^{s}\left(\operatorname{stab}(\delta) ; \mathbb{Z}_{(p)}\right)$ $=0$. So the above spectral sequence converges to

$H^{r}\left(S^{n-2} / \operatorname{Aut}(T, S) ; \mathbb{Z}_{(p)}\right)$.

But another filtration yields a spectral sequence with

$$
E_{2}^{r, s}=H^{r}\left(A u t(T, S) ; H^{s}\left(S^{n-2} ; \mathbb{Z}_{(p)}\right)\right) \Rightarrow H_{\text {Aut }(T, S)}^{r+s}\left(S^{n-2} ; \mathbb{Z}_{(p)}\right)
$$


It follows that $E_{2}^{r, s}=0$ unless $(r, s)$ is $(0,0)$ or $(0, n-2)$. Hence $E_{2}^{0,0}=\mathbb{Z}_{(p)}$ and $E_{2}^{0, n-2}=H^{n-2}\left(S^{n-2} ; \mathbb{Z}_{(p)}\right)^{A u t(T, S)}$. The latter group of invariants is $\mathbb{Z}_{(p)}$ if the action of $\operatorname{Aut}(T, S)$ on $S^{n-2}$ preserves orientation and 0 otherwise. The last assertion in the proposition follows from Corollary 3.2 of [15].

Theorem 3.13. The top dimensional cohomology group of $Q_{\sigma_{n}}$ vanishes. That is, $H^{n-1}\left(Q_{\sigma_{n}} ; \mathbb{Z}_{(p)}\right)=0$.

Proof. We show that the quotient of every maximal cube $(T, S)$ has a free face, so that the interior of the quotient of the cube can be collapsed away. If we can do this, then $Q_{\sigma_{n}}$ will have the same $\mathbb{Z}_{(p)}$-cohomology as an $(n-2)$-dimensional complex, and we will be done.

In the degenerate case where there is an element of $\operatorname{Aut}(T, S)$ which induces an odd permutation of the edges of $S$, then the quotient of the cube $(T, S)$ is not itself a cube. In this case, the diagonal from $(0, \ldots, 0)$ to $(1, \ldots, 1)$ is exposed in the quotient, and any $(n-2)$-dimensional simplex in the quotient which lies next to the diagonal is a free face.

In the nondegenerate case, the quotient of the cube $(T, S)$ is itself a cube, although its boundary might be self indentified in various ways. Since the subforest $S$ of $T$ is maximal, $S$ must contain at least one terminal edge $e$. That is, one of the two vertices of $e$ is a valence 1 vertex or attaching point. Let $\tilde{\Gamma}$ be the graph obtained from $\Gamma=\Gamma_{T}$ by collapsing the subforest $\left\{e, \sigma_{n}(e)\right\}$. The graph $\tilde{\Gamma}$ has a maximal subforest corresponding to collapsing the edges $e$ and $\sigma_{n}(e)$ from the forest $S \cup \sigma_{n}(S)$ of $\Gamma$. Hence we see that collapsing $e$ gives us a face, which we will denote by $(T / e, S / e)$, of the cube $(T, S)$. It can be shown that this face corresponds to a (nondegenerate, cubical) face of the quotient of the cube $(T, S)$ because

Claim 3.14. There is an natural injection of $A u t(\tilde{\Gamma})=\left\langle\sigma_{n}\right\rangle \times \operatorname{Aut}(T / e)$ into $\operatorname{Aut}(\Gamma)=\left\langle\sigma_{n}\right\rangle \times \operatorname{Aut}(T)$. Define the lift $\hat{\phi}$ of an automorphism $\phi \in \operatorname{Aut}(T / e)$ by sending an edge $f$ to $\phi(f)$ if $f \neq e$ and letting $\hat{\phi}(e)=e$.

Proof. Denote by $v$ the valence 1 vertex of $e \in T$ (the attaching point) and let $w$ be the other vertex of $e$. In $T / e, w=v$. We must show that $\phi$ sends $w$ to $w$. This follows automatically, however, as $w=v=\sigma_{n}(w)=\sigma_{n}(v)$ is the only valence 4 vertex of $\tilde{\Gamma}$ and so any automorphism of the graph must fix it. Let $f$ and $g$ be the two other edges in $T$ which share the vertex $w$. Now if $v=*$ then $\phi$ could possibly exchange $f$ and $g$, but this is fine as the lift $\hat{\phi}$ also can. If $v \neq *$, then one of $f$ or $g$ must be closer to the basepoint, and so $\phi$ must fix both $f$ and $g$. Regardless, $\hat{\phi}$ can be defined as in the statement of the claim.

Warning: Note that if $e$ is not a terminal edge, the above claim is false. Collapsing an interior edge somtimes allows you to construct automorphisms with 3-torsion, which obviously cannot be lifted to $\operatorname{Aut}(T)$. 
No automorphism $\phi$ of $(T / e, S / e)$ can induce an odd permutation of the edges in $S / e$, else the lift $\hat{\phi}$ of $\phi$ to $T$ would induce an odd permutation of the edge of $S$. Since $A u t(T)$ is all 2-torsion, it follows from the above claim that $A u t(T / e)$ is also all 2-torsion. Hence the same spectral sequence argument used in Proposition 3.10 yields that the quotient of the cube corresponding to $(T / e, S / e)$ actually is a $\mathbb{Z}_{(p)}$-cohomology cube.

It remains to be shown that the cubical face corresponding to $(T / e, S / e)$ is free. First, if another subforest $S^{\prime}$ with an edge $e^{\prime}$ of $T$ gives a cube with a face isomorphic to $(S / e, S / e)$, then $e^{\prime}$ must also be a terminal edge of $S^{\prime}$. Hence the isomorphism $(T / e, S / e) \rightarrow\left(T / e^{\prime}, S^{\prime} / e^{\prime}\right)$ maps the vertex that $e$ collapsed into to the the vertex that $e^{\prime}$ collapsed into, and so we can lift the isomorphism to one from $(T, S) \rightarrow\left(T, S^{\prime}\right)$.

Second, we must show that blowing up the vertex $w$ in $\tilde{\Gamma}$ only yields graphs isomorphic to $\Gamma$. This follows by considering the ways that the vertex $w$ in $\tilde{\Gamma}$ can be blown up. Say that the edges $f, g, \sigma_{n}(f)$, and $\sigma_{n}(g)$ are the ones incident to $w$. If the ideal edge orbit $\sigma_{n}\{f, g\}$ is blown up, we get back $\Gamma$ exactly, and if $\sigma_{n}\left\{f, \sigma_{n}(g)\right\}$ is blown up, we get a graph isomorphic to $\Gamma$. As these are the only ways to blow up the graph $\sigma_{n}$-equivariantly into another essential graph, we are done.

Corollary 3.15. $H^{n-1}\left(Q_{\sigma_{n}} ; \mathbb{Q}\right)=H^{n-1}\left(\Pi A\left(F_{n}\right) ; \mathbb{Q}\right)=0$.

Proof. That $H^{n-1}\left(Q_{\sigma_{n}} ; \mathbb{Q}\right)=0$ follows immediately from Theorem 3.13. Recall that $\Pi A\left(F_{n}\right)$ acts with finite stabilizers and finite quotient $Q_{\sigma_{n}}$ on the contractible space $L_{\sigma_{n}}$. Since the stabilizers are finite, their rational cohomology vanishes, and the standard equivariant spectral sequence yields that $H^{*}\left(Q_{\sigma_{n}} ; \mathbb{Q}\right)=$ $H^{*}\left(\Pi A\left(F_{n}\right) ; \mathbb{Q}\right)$.

Note that part d) of Theorem 1.1 follows from the above Corollary.

As a final remark for this section, we show that $L_{\sigma_{n}}$ is an $\underline{E} \Pi A\left(F_{n}\right)$ (cf. [19]); that is, for finite subgroups $G$ of $\Pi A\left(F_{n}\right)$, the fixed point subcomplex $L_{\sigma_{n}}^{G}$ is contractible. This follows directly from the corresponding property of $\operatorname{Aut}\left(F_{n}\right)$. The following proposition is unneccesary in the specific case of $L_{\sigma_{n}}$, since (proof omitted) $L_{\sigma_{n}}$ actually equals $X_{n}^{\sigma_{n}}$. This does not normally happen (for example, the spaces $L_{P_{n} \times \sigma_{n}}$ mentioned later in Fact 5.4 are not equal to the corresponding fixed point space of $X_{n}$ ), however, and thus it seems worth noting the more general fact.

Proposition 3.16. Let $S$ be a finite subgroup of $\operatorname{Aut}\left(F_{n}\right)$ and let $\mathcal{S}$ be either $C_{\text {Aut }\left(F_{n}\right)}(S)$ or $N_{\text {Aut }\left(F_{n}\right)}(S)$. Let $L_{S}$ be the retract, defined by Krstic and Vogtmann and consisting of essential marked graphs, of the fixed point subcomplex $X_{n}^{S}$ of the spine of auter space $X_{n}$. Then $L_{S}$ is an $\underline{E} \mathcal{S}$ space. 
Sketch of Proof. Let $H$ be a finite subgroup of $\mathcal{S}$ and let $G$ be the (finite, because $H S H^{-1}=S$ ) subgroup generated by $H$ and $S$. Then $X_{n}^{G}=\left(X_{n}^{S}\right)^{H}=\left(X_{n}^{H}\right)^{S}$, and $X_{n}^{G}$ is contractible from [17]. It remains to be shown that $X_{n}^{G}=\left(X_{n}^{S}\right)^{H}$ deformation retracts to $\left(L_{S}\right)^{H}$.

Given a marked graph $\Gamma$ representing a vertex of $\left(X_{n}^{S}\right)^{H}$, we must show (see Proposition 3.3 of [21]) that for every edge $e$ in $\Gamma$ and every $h \in H, e$ is $S$-inessential if and only if he is $S$-inessential. This follows automatically from Corollary 4.5 of [21], which characterizes essential edges by looking at the stabilizers (in $S$ ) of paths in $\Gamma$. Since $H S H^{-1}=H$, the stabilizers in $h$-translates of such paths are still in $S$ and are isomorphic (conjugate by $h$ ) to those of the original path.

\section{A realization proposition}

Let $\hat{A}$ be a finite subgroup of $\Pi A\left(F_{n}\right)$ and let $A$ be the (finite) subgroup generated by $\hat{A}$ and $\sigma_{n}$. By Zimmerman's [22] realization theorem, we can realize $A$ by an action on an $A$-reduced graph $\Gamma$. From the proposition below, $\Gamma$ is also $\left\langle\sigma_{n}\right\rangle-$ reduced; that is, $\Gamma$ is a $\theta_{1}$-tree.

Note that the corresponding statement is not true in $\operatorname{Out}\left(F_{n}\right)$ (have $\mathbb{Z} / p \times$ $\left\langle\sigma_{p-1}\right\rangle$ act on a $\theta$-graph $\left.\theta_{p-1}\right)$ and certainly would not be true in $\operatorname{Aut}\left(F_{n}\right)$ if the $\sigma_{n}$-action were replaced by some other $\mathbb{Z} / 2$-action.

Proposition 4.1. Let $A \subseteq \Pi A\left(F_{n}\right)$ be a finite subgroup of the palindromic automorphism group with $\sigma_{n} \in A$. Realize $A$ by an action on an A-reduced marked graph $\phi: R_{n} \rightarrow \Gamma$. Then $\phi: R_{n} \rightarrow \Gamma$ is also a $\left\langle\sigma_{n}\right\rangle$-reduced marked graph.

Proof. As before, let $F_{n}=\left\langle a_{1}, \ldots, a_{n}\right\rangle$ and identify the petals of the rose $R_{n}$ with the generators $a_{i}$. Note that $\Gamma$ has no separating edges, else it would not be $A$-reduced. In this proof, when we refer to concepts such as the number of times an edge $e$ of $\Gamma$ occurs in some $\phi\left(a_{i}\right)$, we mean that we should take the unique taut path in $\Gamma$, starting and ending at $*$, which is homotopic to the path $\phi\left(a_{i}\right)$ in $\Gamma$, and then count the number of times $e$ occurs in this taut path. By way of contradiction, suppose $\Gamma$ is not $\left\langle\sigma_{n}\right\rangle$-reduced. Let $e_{1} \in \Gamma$ be an edge of minimal distance to the basepoint $*$ such that $\left\{e_{1}, \sigma_{n} e_{1}\right\}$ is a forest.

CASE 1: $e_{1}=\sigma_{n} e_{1}$. Since $e_{1}$ is not a separating edge of $\Gamma$, we can choose a nontrivial cycle $\mu$, starting and ending at $*$, which has just one occurence of $e_{1}$ and none of $e_{1}^{-1}$. If for all $i=1, \ldots, n$, the cycles $\phi\left(a_{i}\right)$ have an even number of occurences of $e_{1}^{ \pm 1}$, then we could not write $\mu$ as a product of them and their inverses. So some $\phi\left(a_{j}\right)$ has an odd number of occurences of $e_{i}^{ \pm 1}$ in it. Say that the exponent sum of $e_{1}$ in $\phi\left(a_{j}\right)$ is $k, k$ odd. Then the exponent sum of $e_{1}$ in $\sigma_{n} \phi\left(a_{j}\right)$ is still $k$, but the exponent sum of $\phi\left(a_{j}^{-1}\right)$ is $-k$. This contradicts the fact that $\sigma_{n} a_{j}=a_{j}^{-1}$.

$C A S E$ 2: $\quad e_{1} \neq \sigma_{n} e_{1}$. Let $\alpha$ be a shortest length path from $*$ to $e_{1}$. Say without 
loss of generality that $e_{1}$ is the oriented edge from $v$ to $w$ and that $\alpha$ goes from $*$ to $v$. Let $f_{1}=\sigma_{n} e_{1}$. Then $\sigma_{n} \alpha$ is a shortest length path from $*$ to $f_{1}$. Now $v=\sigma_{n} v$, else we could write $\alpha=\beta b$ and get $\left\{b, \sigma_{n} b\right\}$ as a $\sigma_{n}$-invariant forest closer to $*$ than $\left\{e_{1}, f_{1}\right\}$ is. (If $|\alpha|=0$, then $v=*$ and so $\sigma_{n}(v)=v$ necessarily.) So we have both $\alpha$ and $\sigma_{n} \alpha$ are paths from $*$ to $v$ Moreover, $w \neq \sigma_{n} w$ (else $\left\{e_{1}, \sigma_{n} e_{1}\right\}$ is not a forest.) Now $A e_{1}=A\left\{e_{1}, f_{1}\right\}$ is not a forest, since $\Gamma$ is $A$-reduced. Hence we can choose some simple closed curve $\mu$ in $A e_{1} \subseteq \Gamma$ that contains $e_{1}$. There must exist some $a e_{1}^{ \pm 1} \in \mu, a e_{1}^{ \pm 1} \notin\left\{e_{1}, e_{1}^{-1}\right\}$, such that $a w=w$. Why? Otherwise we could deformation retract $\mu$ to the set of vertices $\left\{\hat{a} v: \hat{a} e_{1}^{ \pm 1} \in \mu\right\}$, which contradicts the fact that $\mu$ is a simple closed curve. Now $a e_{1} \neq f_{1}$, as $\sigma_{n} w \neq w$. Hence $\alpha e_{1}\left(a e_{1}\right)^{-1}(a \alpha)^{-1}$ is a nontrivial cycle starting and ending at $*$ which contains exactly one occurence of $e_{1}$ and none of $f_{1}$. So there must be a $\phi\left(a_{j}^{\epsilon}\right), \epsilon \in\{-1,1\}$, which contains an odd number of occurences of $e_{1}^{ \pm 1}$ and an even number of occurences of $f_{1}^{ \pm 1}$. (If we had some even/odd $\phi\left(a_{j}\right)$, then we could act by $\sigma_{n}$ to get odd/even, and this would be a $\phi\left(a_{j}^{-1}\right)$. Otherwise, all $\phi\left(a_{i}\right)$ are all even/even or odd/odd, and so combine together just to get more even/even or odd/odd loops.) This is a contradiction, however, because $\phi\left(a_{j}^{-\epsilon}\right)$ still has an odd number of occurences of $e_{1}^{ \pm 1}$ while $\phi\left(\sigma_{n} a_{j}^{\epsilon}\right)$ has an even number of occurences of $e_{1}^{ \pm 1}$.

Proof of part b) (i) of Theorem 1.1. From the action of $(\mathbb{Z} / 2)^{n}$ on the rose $R_{n}$ such that the $i$ th generator inverts the $i$ th petal and leaves all others fixed, we know that the Krull dimension at the prime 2 is at least $n$. Similarly, there is an action of $(\mathbb{Z} / p)^{\left[\frac{n}{p}\right]}$ on $R_{n}$ where the first $\mathbb{Z} / p$ rotates the first $p$ petals, the second $\mathbb{Z} / p$ rotates the next $p$ petals, etc. Hence the Krull dimension at the prime $p$ is at least $\left[\frac{n}{p}\right]$.

Let $A$ be a maximal rank elementary abelian subgroup of $\Pi A\left(F_{n}\right)$. From Proposition 4.1, we can realize $A$ by an action of $A$ on a $\sigma_{n}$-graph $\Gamma$ which is both $A$-reduced and $\sigma_{n}$-reduced. That is, we have an action of $A$ on a pointed $\Theta_{1}$-tree $\Gamma$. Since elements of $A$ must preserve basepoints, the action of $A$ on the tree $\Gamma / \sigma_{n}$ does not invert edges. Hence we have inclusions

$$
A \longmapsto(\mathbb{Z} / 2)^{n} \rtimes A u t_{*}\left(\Gamma / \sigma_{n}\right) \longmapsto(\mathbb{Z} / 2)^{n} \rtimes \Sigma_{n}=\mathbb{Z} / 2 \prec \Sigma_{n} .
$$

The result (for 2 or odd primes $p$ ) now follows from standard facts about $\Sigma_{n}$ (cf. Theorem 1.3 in Chapter VI of [1].)

Proof of part c) of Theorem 1.1. We sketch the proof, which uses standard methods. Suppose that some $A=\mathbb{Z} / p$ lies in $E \Pi A\left(F_{n}\right)$. From Proposition 4.1, we can realize $A$ by an action of $A$ on a $\sigma_{n}$-graph $\Gamma$ which is both $A$-reduced and $\sigma_{n}$-reduced. Let $\phi: R_{n} \rightarrow \Gamma$ be the corresponding marked graph. Let $T=\Gamma / \sigma_{n}$, a pointed tree with an $A$-action on it. First, suppose that the $A$-action on $T$ is 
nontrivial. (This will always be the case if $p$ is odd.) Then there are two edges $e_{1}$ and $e_{2}$, both oriented so that their terminal vertices are closer to the basepoint than their initial vertices, of $\Gamma$ such that a generator of $A$ rotates the edge $\left[e_{1}\right]$ into the edge $\left[e_{2}\right]$ in $T$. Some generator $a_{i_{1}}$ of $F_{n}$ must be such that $\phi\left(a_{i_{1}}\right)$ contains an odd number of occurences of $e_{1}$ in it. Choose $a_{i_{2}}$ similarly. Then $\phi\left(a_{i_{j}}\right)$ is a palindromic word in the edges of $\Gamma$ with either $\bar{e}_{i_{j}} \sigma_{n}\left(e_{i_{j}}\right)$ or $\sigma_{n}\left(\bar{e}_{i_{j}}\right) e_{i_{j}}$ in the middle of the palindrome. The generator of $A$ (thought of as an element of $\left.E \Pi A\left(F_{n}\right)\right)$ must send $a_{i_{1}}$ to a palindrome with either $a_{i_{2}}$ or $a_{i_{2}}^{-1}$ in the center of it. This contradicts the fact that all elements of $E \Pi A\left(F_{n}\right)$ send generators $a_{i}$ to palidromes with $a_{i}$ in the center of them.

The only remaining case is where $p=2$ and $A$ acts trivally on $T$. So $A$ is a subgroup of the group $(\mathbb{Z} / 2)^{n}$ of graph automorphisms of $\Gamma$ which act by inverting the $\Theta_{1}$ 's in the $\Theta_{1}$-tree $\Gamma$. Hence the generator of $A$ corresponds to an element of $\Pi A\left(F_{n}\right)$ which, for at least one $i$, sends $a_{i}$ to a palindrome with $a_{i}^{-1}$ in its center. As none of these automorphisms are in $E \Pi A\left(F_{n}\right)$, we again have a contradiction.

\section{Cohomology of $\Pi A\left(F_{n}\right)$ at odd primes $p$}

Let $p$ be an odd prime (as will always be the case from now on in this paper.) We wish to calculate the Farrell cohomology of $\Pi A\left(F_{n}\right)$ using Ken Brown's [2] normalizer spectral sequence, which states that

$$
E_{1}^{r, s}=\prod_{\left(P_{0} \subset \cdots \subset P_{r}\right) \in|\mathcal{B}|_{r}} \hat{H}^{s}\left(\bigcap_{i=0}^{r} N_{G}\left(P_{i}\right) ; \mathbb{Z}_{(p)}\right) \Rightarrow \hat{H}^{r+s}\left(G ; \mathbb{Z}_{(p)}\right)
$$

where $G$ is a group with finite virtual cohomological dimension, $\mathcal{A}$ is the poset of nontrivial elementary abelian $p$-subgroups of $G, \mathcal{B}$ is the poset of conjugacy classes of nontrivial elementary abelian $p$-subgroups of $G$, and $|\mathcal{B}|_{r}$ is the set of $r$-simplices in the realization $|\mathcal{B}|$.

A first step toward performing such a calculation is calculating $|\mathcal{B}|$. In other words, we wish to calculate conjugacy classes of elementary abelian subgroups $P \subset \Pi A\left(F_{n}\right)$. By Proposition 4.1, we can realize such finite groups $P$ by reduced actions on $\theta_{1}$-trees.

If $n \geq p$, define a particular subgroup $P_{n} \cong \mathbb{Z} / p$ of $\Pi A\left(F_{n}\right)$ by letting $P_{n}$ act on the rose $R_{n}$ by rotating its first $p$ leaves and leaving the last $n-p$ leaves fixed. That is, $P_{n}$ corresponds to automorphisms which rotate the first $p$ generators $a_{1}, \ldots, a_{p}$ and leave the remaining generators fixed.

Corollary 5.2. If $p \leq n \leq 2 p-1$, then

$$
\hat{H}^{*}\left(\Pi A\left(F_{n}\right) ; \mathbb{Z}_{(p)}\right) \cong \hat{H}^{*}\left(N_{A u t\left(F_{n}\right)}\left(P_{n} \times\left\langle\sigma_{n}\right\rangle\right) ; \mathbb{Z}_{(p)}\right) .
$$


Proof. We show that $P_{n}$ is the only conjugacy class of nontrivial elementary abelian $p$-subgroups that is in $\Pi A\left(F_{n}\right)$. By Proposition 4.1, we see that an arbitrary nontrivial elementary abelian $p$-subgroup $A$ comes from some action on a $\theta_{1}$-tree with $p$-symmetry. Since $p \leq n \leq 2 p-1$, the only possibility is that $A$ acts on a $\theta_{1}$-tree $\Gamma$ by rotating $p$ of the $\theta_{1}$-leaves and leaving the other $n-p \theta_{1}$-edges in the tree fixed. But it is clear that a product of $\left(P_{n} \times\left\langle\sigma_{n}\right\rangle\right)$-Nielsen transformations takes the rose $R_{n}$ to the graph $\Gamma$, and hence we see that $A$ and $P_{n}$ are conjugate to each other in $\Pi A\left(F_{n}\right)$.

By the normalizer spectral sequence 5.1 , this yields that

$$
\hat{H}^{*}\left(\Pi A\left(F_{n}\right) ; \mathbb{Z}_{(p)}\right) \cong \hat{H}^{*}\left(N_{\Pi A\left(F_{n}\right)}\left(P_{n}\right) ; \mathbb{Z}_{(p)}\right) .
$$

But since $p$ is an odd prime, it is easy to see that

$$
N_{\Pi A\left(F_{n}\right)}\left(P_{n}\right)=N_{A u t\left(F_{n}\right)}\left(P_{n} \times\left\langle\sigma_{n}\right\rangle\right) .
$$

\section{Proposition 5.3.}

$$
N_{A u t\left(F_{n}\right)}\left(P_{n} \times\left\langle\sigma_{n}\right\rangle\right) \cong N_{\Sigma_{p}}\left(P_{n}\right) \times\left(F_{m} \rtimes\left(\left\langle\sigma_{p}\right\rangle \times \Pi A\left(F_{m}\right)\right)\right)
$$

where $m=n-p, \Pi A\left(F_{m}\right)$ acts on the $F_{m}$ in the semidirect product in the natural way, and $\sigma_{p}$ acts on $F_{m}$ as $\sigma_{m}$ does.

Proof. The $N_{\Sigma_{p}}\left(P_{n}\right)$ in the above decomposition comes from automorphisms of $F_{n}$ which permute the first $p$ generators and leave the remaining $m$ fixed. The $F_{m}$ being acted upon in the semidirect product structure above has $i$ th generator $\left(a_{1} \| a_{p+i}\right)\left(a_{2} \| a_{p+i}\right) \ldots\left(a_{p} \| a_{p+i}\right)$. The $\sigma_{p}$ is the involution which inverts the first $p$ generators of $F_{n}$ and leaves the remaining $m$ fixed. Finally, the $\Pi A\left(F_{m}\right)$ comes from automorphisms which fix the first $p$ generators of $F_{n}$ and act on the last $m$ generators by identifying the subgroup $\left\langle a_{p+1}, a_{p+2}, \ldots, a_{n}\right\rangle$ with $F_{m}$.

Consider the action of $P_{n} \times\left\langle\sigma_{n}\right\rangle$ on the rose $R_{n}$. $P_{n}$ rotates the first $p$ petals. Label the first $p$ petals of the rose as $a_{1}, \ldots, a_{p}$ as before, but label the last $m$ petals as $b_{1}, \ldots, b_{m}$.

Since $\left|P_{n}\right|=p$ is an odd prime, $N_{A u t\left(F_{n}\right)}\left(P_{n} \times\left\langle\sigma_{n}\right\rangle\right) \subseteq N_{A u t\left(F_{n}\right)}\left(P_{n}\right)$ and in Lemma 5.1 of [16], we calculated

$$
N_{A u t\left(F_{n}\right)}\left(P_{n}\right) \cong N_{\Sigma_{p}}\left(P_{n}\right) \times\left(\left(F_{m} \times F_{m}\right) \rtimes\left(\left\langle\sigma_{p}\right\rangle \times \operatorname{Aut}\left(F_{m}\right)\right)\right),
$$

where the first $F_{m}$ in $F_{m} \times F_{m}$ is the free group on the $P_{n}$-Nielsen transformations $\left\langle a_{1}, b_{i}^{-1}\right\rangle$ for $i \in\{1, \ldots, m\}$ and the latter $F_{m}$ is the free group on the $P_{n}$-Nielsen transformations $\left\langle a_{1}^{-1}, b_{i}^{-1}\right\rangle, i \in\{1, \ldots, m\}$. Note that $\left\langle\sigma_{p}\right\rangle$ acts on $F_{m} \times F_{m}$ via $\sigma_{p}\left\langle a_{1}, b_{i}^{-1}\right\rangle \sigma_{p}=\left\langle a_{1}^{-1}, b_{i}^{-1}\right\rangle$ and $\sigma_{p}\left\langle a_{1}^{-1}, b_{i}^{-1}\right\rangle \sigma_{p}=\left\langle a_{1}, b_{i}^{-1}\right\rangle$. In other words, if $(b, c) \in F_{m} \times F_{m}$ then $\sigma_{p}(b, c) \sigma_{p}=(c, b)$. 
Let $G$ be the subgroup

$$
N_{\Sigma_{p}}\left(P_{n}\right) \times\left(F_{m} \rtimes\left(\left\langle\sigma_{p}\right\rangle \times C_{A u t\left(F_{m}\right)}\left(\sigma_{m}\right)\right)\right)
$$

of $N_{A u t\left(F_{n}\right)}\left(P_{n}\right)$, where $F_{m}$ is the free group on the generators $\left\langle a_{1}, b_{i}\right\rangle \circ\left\langle a_{1}^{-1}, b_{i}^{-1}\right\rangle$ for $i \in\{1, \ldots, m\}$, and $C_{A u t\left(F_{m}\right)}\left(\sigma_{m}\right)$ is included in $\operatorname{Aut}\left(F_{m}\right)$ in the obvious way. It follows directly that $G \subseteq N_{A u t\left(F_{n}\right)}\left(P_{n} \times\left\langle\sigma_{n}\right\rangle\right)$. To prove the proposition, we must show that they are equal.

Take an arbitrary

$$
x \in N_{A u t\left(F_{n}\right)}\left(P_{n} \times\left\langle\sigma_{n}\right\rangle\right) \subseteq N_{\Sigma_{p}}\left(P_{n}\right) \times\left(\left(F_{m} \times F_{m}\right) \rtimes\left(\left\langle\sigma_{p}\right\rangle \times A u t\left(F_{m}\right)\right)\right) .
$$

Say $x=a b c d e$, where $a \in N_{\Sigma_{p}}\left(P_{n}\right),(b, c) \in F_{m} \times F_{m}, d \in\left\langle\sigma_{p}\right\rangle$, and $e \in \operatorname{Aut}\left(F_{m}\right)$. Since $a, d \in N_{\text {Aut }\left(F_{n}\right)}\left(P_{n} \times\left\langle\sigma_{n}\right\rangle\right), a^{-1} x d^{-1}=b c e \in N_{A u t\left(F_{n}\right)}\left(P_{n} \times\left\langle\sigma_{n}\right\rangle\right)$ also. So $b c e \in \Pi A\left(F_{n}\right)$ and $(b c e) \sigma_{n}(b c e)^{-1}=\sigma_{n}$. This means that the map $(b c e) \sigma_{n}(b c e)^{-1}$ sends $a_{i}$ to $a_{i}^{-1}$ for $i \in\{1, \ldots, p\}$ and $b_{i}$ to $b_{i}^{-1}$ for $i \in\{1, \ldots, m\}$. Now both $\sigma_{n}$ and $e$ restrict to maps in $A u t\left(\left\langle b_{1}, \ldots, b_{m}\right\rangle\right)$ and moreover $b$ and $c$ both restrict to the identity map in $\operatorname{Aut}\left(\left\langle b_{1}, \ldots, b_{m}\right\rangle\right)$. Hence for $i \in\{1, \ldots, m\}$, we have

$$
b_{i}^{-1}=(b c e) \sigma_{n}(b c e)^{-1}\left(b_{i}\right)=e \sigma_{n} e^{-1}\left(b_{i}\right),
$$

and we see that $e \sigma_{n} e^{-1}$ restricts to $\sigma_{m}$ in $\operatorname{Aut}\left(F_{m}\right)$. As $e \in A u t\left(F_{m}\right)$, this means $e \in C_{A u t\left(F_{m}\right)}\left(\sigma_{m}\right)$. Hence $e \in \Pi A\left(F_{n}\right)$ also. Since bce $\in \Pi A\left(F_{n}\right)$, this gives $b c \in \Pi A\left(F_{n}\right)$. In other words, we have

$$
(b, c) \in\left(F_{m} \times F_{m}\right) \subseteq N_{\Sigma_{p}}\left(P_{n}\right) \times\left(\left(F_{m} \times F_{m}\right) \rtimes\left(\left\langle\sigma_{p}\right\rangle \times \operatorname{Aut}\left(F_{m}\right)\right)\right)
$$

and

$$
(b, c) \in \Pi A\left(F_{n}\right) .
$$

It follows that

$$
\begin{aligned}
(b, c) & =\sigma_{n}(b, c) \sigma_{n} \\
& =\sigma_{m} \sigma_{p}(b, c) \sigma_{p} \sigma_{m} \\
& =\sigma_{m}(c, b) \sigma_{m} \\
& =\left(\sigma_{m}(c), \sigma_{m}(b)\right) .
\end{aligned}
$$

So $b=\sigma_{m}(c)$ and $c=\sigma_{m}(b)$. In summary, we have shown that an arbitrary element $x=a b c d e \in N_{A u t\left(F_{n}\right)}\left(P_{n} \times\left\langle\sigma_{n}\right\rangle\right)$ has $c=\sigma_{m}(b)$ and $e \in C_{A u t\left(F_{m}\right)}\left(\sigma_{m}\right)$. Thus $x \in G$, as desired.

The group $N_{\text {Aut }\left(F_{n}\right)}\left(P_{n} \times\left\langle\sigma_{n}\right\rangle\right)$ acts on the contractible space $L_{P_{n} \times\left\langle\sigma_{n}\right\rangle}$ with finite stabilizers and finite quotient $Q_{P_{n} \times\left\langle\sigma_{n}\right\rangle}=L_{P_{n} \times\left\langle\sigma_{n}\right\rangle} / N_{A u t\left(F_{n}\right)}\left(P_{n} \times\left\langle\sigma_{n}\right\rangle\right)$. 
Define a $p$-admissible tree $T$ to be a triple $(T, \circ, A)$ where $T$ is a pointed tree, $\circ$ is a vertex of $T$ (which may be the basepoint $*$ ), $A$ is a subset of the vertices of $T$ called the set of attaching points, $* \in A$, and all valence 1 vertices of $T$ are in $A$. For a $p$-admissible tree $T$, define the corresponding graph $\Gamma_{T}$ as follows: Take two isomorphic copies $T_{1}$ and $T_{2}$ of the tree $T$, and let $f: T_{1} \rightarrow T_{2}$ be an isomorphism. Then let $\Gamma_{T}^{\text {pre }}$ be the graph

$$
\Gamma_{T}^{p r e}=\frac{T_{1} \amalg T_{2}}{f(v) \sim v, \text { for all attaching points } v \text { in } A .}
$$

Let $\theta_{p-1}$ be a $\theta$-graph with $p$ edges and two vertices $v_{1}$ and $v_{2}$. Let $\circ_{1}$ be the o-vertex in $T_{1}$ and let $\circ_{2}=f\left(\circ_{1}\right)$ be the o-vertex in $T_{2}$. Finally, let

$$
\Gamma_{T}=\frac{\Gamma_{T}^{p r e} \amalg \theta_{p-1}}{\circ_{1} \sim v_{1}, \circ_{2} \sim v_{2}}
$$

If $\pi_{1}\left(\Gamma_{T}\right) \cong F_{n}$, then say $T$ is a $p$-admissible tree of rank $n$.

If $T$ is a $p$-admissible tree of rank $n$, define a $\left\langle\sigma_{n}\right\rangle$-action on the edges of $\Gamma_{T}$ by

$$
\sigma_{n} x= \begin{cases}f(x), & x \in T_{1} \\ f^{-1}(x), & x \in T_{2} \\ x^{-1}, & x \in \theta_{p-1}\end{cases}
$$

Since this action inverts the edges of the $\theta$-graph in $\Gamma_{T}$, we then need to subdivide these edges so that the group acts without inversions. Next, define a $P_{n}$-action on $\Gamma_{T}$ by having $P_{n}$ fix $\Gamma_{T}^{\text {pre }}$ and rotate the edges of $\theta_{p-1}$ cyclically. In this way, $\Gamma_{T}$ is a $\left(P_{n} \times\left\langle\sigma_{n}\right\rangle\right)$-graph.

A $p$-admissible tree is $T$ reduced if the corresponding $\left(P_{n} \times\left\langle\sigma_{n}\right\rangle\right)$-graph $\Gamma_{T}$ is reduced; that is, if all vertices of $T$ are attaching points. Similarly, a $p$-admissible tree $T$ is a maximal if the attaching points of $T$ are exactly its valence 1 vertices, the valence 2 vertices of $T$ consist of just the point $\circ$, and $T$ has no vertices with valence 4 or more. As before, a subforest of $T$ is a collection of edges $S$ of $T$ such that there is no path in $S$ from one attaching point to another. Lastly, isomorphisms of $p$-admissible trees must be graph isomorphisms which take $*$ to *, $\circ$ to $\circ$, and $A$ to $A$.

The following facts about $\left(P_{n} \times\left\langle\sigma_{n}\right\rangle\right)$-graphs are all proven in similar ways to the analogous facts about $\sigma_{n}$-graphs.

\section{Fact 5.4.}

(1) There is a bijective correspondence between reduced $p$ - admissible trees of rank $n$ and the underlying graphs of $\left(P_{n} \times\left\langle\sigma_{n}\right\rangle\right)$ - reduced marked graphs, given by $T \rightarrow \Gamma_{T}$.

(2) There is a bijective correspondence between maximal p-admissible trees of rank $n$ and the underlying graphs of maximal essential marked $\left(P_{n} \times\left\langle\sigma_{n}\right\rangle\right)$-graphs, given by $T \rightarrow \Gamma_{T}$. 
(3) The virtual cohomological dimension of $N_{\text {Aut }\left(F_{n}\right)}\left(P_{n} \times\left\langle\sigma_{n}\right\rangle\right)$ is $m=n-p$.

(4) Let $\Gamma$ be a graph which occurs as the underlying graph of a marked graph in $L_{P_{n} \times\left\langle\sigma_{n}\right\rangle}$. Then there is only one possible $\sigma_{n}$-action on $\Gamma$.

(5) If two marked graphs in $L_{P_{n} \times\left\langle\sigma_{n}\right\rangle}$ have underlying graphs which correspond to the same $p$-admissible tree, then they correspond to the same vertex in $Q_{P_{n} \times\left\langle\sigma_{n}\right\rangle}$. That is, we can form the moduli space $Q_{P_{n} \times\left\langle\sigma_{n}\right\rangle}$ by looking only at the poset structure of the p-admissible trees corresponding to marked graphs in $L_{P_{n} \times\left\langle\sigma_{n}\right\rangle}$.

(6) The top dimensional cohomology class of $Q_{P_{n} \times\left\langle\sigma_{n}\right\rangle}$, with coefficients in $\mathbb{Z}_{(p)}$, vanishes. That is, $H^{n-p}\left(Q_{P_{n} \times\left\langle\sigma_{n}\right\rangle} ; \mathbb{Z}_{(p)}\right)=0$.

(7) $H^{n-p}\left(Q_{P_{n} \times\left\langle\sigma_{n}\right\rangle} ; \mathbb{Q}\right)=H^{n-p}\left(N_{A u t\left(F_{n}\right)}\left(P_{n} \times\left\langle\sigma_{n}\right\rangle\right) ; \mathbb{Q}\right)=0$.

Note that (4) and (5) above are a little bit different from their analogs Proposition 3.7 and Corollary 3.8. Basically, the underlying graphs $\Gamma$ always have just one possible $\sigma_{n}$-action, as before, but it is conceivable (for example, if the graph contains two or more copies of $\theta_{p-1}$ inside it and we must decide which one $P_{n}$ rotates) that it might have several possible $P_{n}$-actions. That is why we talk about $p$-admissible trees instead in (5), since the vertex $\circ$ in the tree determines where the $p$ edges that $P_{n}$ rotates are located.

Fact 5.4 allows us to show

Proposition 5.5. If $p \leq n \leq 2 p-1$, then

$$
\hat{H}^{t}\left(N_{\text {Aut }\left(F_{n}\right)}\left(P_{n} \times\left\langle\sigma_{n}\right\rangle\right) ; \mathbb{Z}_{(p)}\right) \cong \begin{cases}\mathbb{Z} / p & t \equiv 0(\bmod 2(p-1)) \\ H^{r}\left(Q_{P_{n} \times\left\langle\sigma_{n}\right\rangle} ; \mathbb{Z} / p\right) & t \equiv r(\bmod 2(p-1)), \\ & 1 \leq r \leq n-p-1 \\ 0 & t \equiv r(\bmod 2(p-1)), \\ & n-p \leq r \leq 2 p-3\end{cases}
$$

Proof. We use the equivariant cohomology spectral sequence for $N_{A u t\left(F_{n}\right)}\left(P_{n} \times\left\langle\sigma_{n}\right\rangle\right)$ acting on the contractible space $L_{P_{n} \times\left\langle\sigma_{n}\right\rangle}$ with finite stabilizers and finite quotient $Q_{P_{n} \times\left\langle\sigma_{n}\right\rangle}$. The equivariant cohomology spectral sequence for this action is

$$
\begin{array}{r}
E_{1}^{r, s}=\prod_{[\delta] \in \Delta_{n}^{r}} \hat{H}^{s}\left(\operatorname{stab}_{N_{A u t\left(F_{n}\right)}\left(P_{n} \times\left\langle\sigma_{n}\right\rangle\right)}(\delta) ; \mathbb{Z}_{(p)}\right) \\
\left.\Rightarrow \hat{H}^{r+s}\left(N_{A u t\left(F_{n}\right)}\left(P_{n} \times\left\langle\sigma_{n}\right\rangle\right)\right) ; \mathbb{Z}_{(p)}\right)
\end{array}
$$

where $[\delta]$ ranges over the set $\Delta_{n}^{r}$ of orbits of $r$-simplices $\delta$ in $\left.L_{P_{n} \times\left\langle\sigma_{n}\right.}\right\rangle$.

From the decomposition

$$
N_{\text {Aut }\left(F_{n}\right)}\left(P_{n} \times\left\langle\sigma_{n}\right\rangle\right) \cong N_{\Sigma_{p}}\left(P_{n}\right) \times\left(F_{m} \rtimes\left(\left\langle\sigma_{p}\right\rangle \times C_{\text {Aut }\left(F_{m}\right)}\left(\sigma_{m}\right)\right)\right)
$$

we see that $\left(F_{m} \rtimes\left(\left\langle\sigma_{p}\right\rangle \times C_{A u t\left(F_{m}\right)}\left(\sigma_{m}\right)\right)\right)$ has $p$-rank 0 . Since $N_{\Sigma_{p}}\left(P_{n}\right)$ acts trivially on marked graphs in $L_{P_{n} \times\left\langle\sigma_{n}\right\rangle}$ by permuting the edges of the $\theta$-graph 
attached at $\circ$, it follows that for every simplex $\delta$ we have

$$
\hat{H}^{*}\left(\operatorname{stab}_{N_{A u t\left(F_{n}\right)}\left(P_{n} \times\left\langle\sigma_{n}\right\rangle\right)}(\delta) ; \mathbb{Z}_{(p)}\right) \cong \hat{H}^{*}\left(N_{\Sigma_{p}}\left(P_{n}\right) ; \mathbb{Z}_{(p)}\right) \cong \hat{H}^{*}\left(\Sigma_{p} ; \mathbb{Z}_{(p)}\right) .
$$

The $E_{1}^{r, s}$-page of the spectral sequence is 0 in the rows where $s \neq k \cdot 2(p-1)$ and a copy of the cellular cochain complex with $\mathbb{Z} / p$-coefficients of the $(n-p)$ dimensional complex $Q_{P_{n} \times\left\langle\sigma_{n}\right\rangle}$ in rows $k \cdot 2(p-1)$. It follows that the $E_{2}$-page has the form:

$$
E_{2}^{r, s}= \begin{cases}\mathbb{Z} / p & r=0 \text { and } s=k \cdot 2(p-1) \\ H^{r}\left(Q_{P_{n} \times\left\langle\sigma_{n}\right\rangle} ; \mathbb{Z} / p\right) & 1 \leq r \leq n-p \text { and } s=k \cdot 2(p-1) \\ 0 & \text { otherwise }\end{cases}
$$

Hence we see that the spectral sequence converges at the $E_{2}$-page.

That $H^{n-p}\left(Q_{P_{n} \times\left\langle\sigma_{n}\right\rangle} ; \mathbb{Z} / p\right)=0$ follows from part 6 of Fact 5.4 and universal coefficients.

Note that the above proposition immediately proves part b) (ii) of Theorem 1.1 .

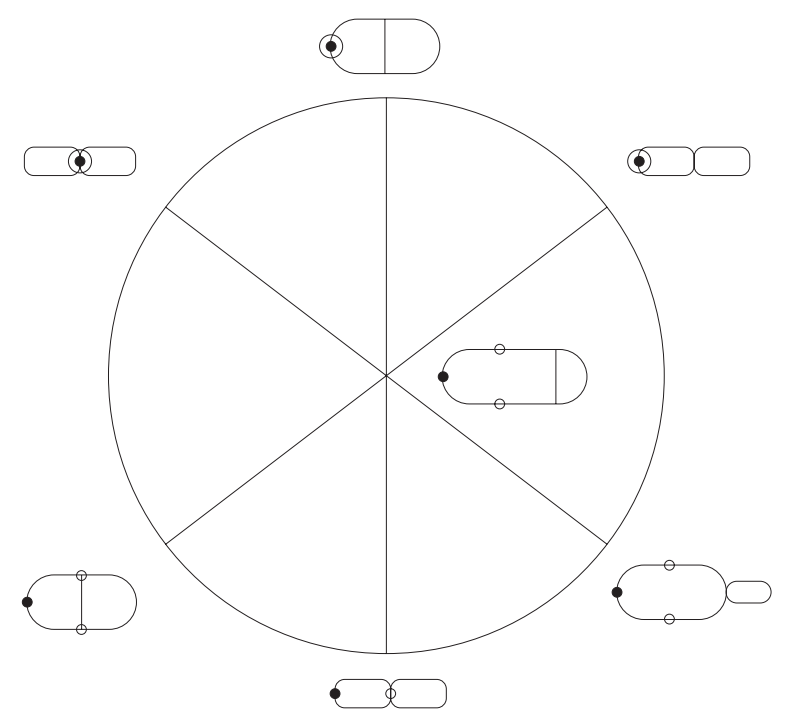

Figure 1. Simplices from the first maximal graph

By examining the space $Q_{P_{n} \times\left\langle\sigma_{n}\right\rangle}$ in low dimensions where $m \in\{0,1,2\}$ and showing that it is contractible, we have the following corollary, which will give us part e) of Theorem 1.1: 
Corollary 5.6. If $m=n-p \in\{0,1,2\}$, then

$$
\hat{H}^{*}\left(\Pi A\left(F_{n}\right) ; \mathbb{Z}_{(p)}\right) \cong \hat{H}^{*}\left(N_{A u t\left(F_{n}\right)}\left(P_{n} \times\left\langle\sigma_{n}\right\rangle\right) ; \mathbb{Z}_{(p)}\right) \cong \hat{H}^{*}\left(\Sigma_{p} ; \mathbb{Z}_{(p)}\right) .
$$

Proof. CASE 1: $m=0$. Then $Q_{P_{n} \times\left\langle\sigma_{n}\right\rangle}$ is a point.

CASE 2: $m=1$. Then $Q_{P_{n} \times\left\langle\sigma_{n}\right\rangle}$ is a contractible 1-dimensional complex with 3 vertices and two edges. Define the maximal $p$-admissible tree $T$ of rank $n$ to be the tree with three vertices $*, \circ, v$ and two edges $e_{1}, e_{2}$ where $e_{1}$ goes from $*$ to $\circ$ and $e_{2}$ goes from $\circ$ to $v$. The middle vertex of the 1-dimensional complex $Q_{P_{n} \times\left\langle\sigma_{n}\right\rangle}$ corresponds to the graph $\Gamma_{T}$. The other two vertices and two edges $Q_{P_{n} \times\left\langle\sigma_{n}\right\rangle}$ correspond to the two possible ways that $\Gamma_{T}$ can be collapsed equivariantly.

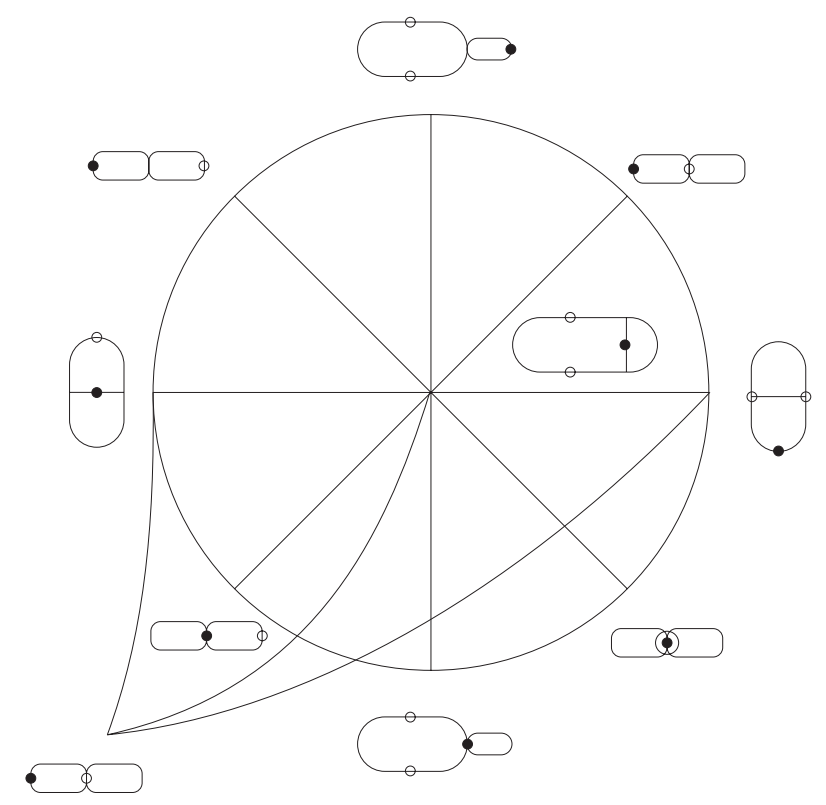

Figure 2. Simplices from the second maximal graph

CASE 3: $\mathrm{m}=2$. Then $Q_{P_{n} \times\left\langle\sigma_{n}\right\rangle}$ is a 2-dimensional complex with 13 vertices, 28 edges, and 16 two-simplices. There are two maximal graphs in $Q_{P_{n} \times\left\langle\sigma_{n}\right\rangle}$. Simplices coming from the first graph are listed in figure 1 and simplices from the second graph are listed in figure 2. In figures 1 and 2, the maximal graphs are listed in the center. These maximal graphs can be collapsed in various ways, and these are listed around the periphery of the figures. In the graphs, a solid dot indicates the basepoint $*$ and the hollow dots represent attaching points $\circ$ for the $\theta$-graph $\theta_{p-1}$. If there is only one hollow dot in a graph, both ends of the $\theta$-graph should be attached to that one vertex. Upon identifying the boundaries of the simplices 
listed in figures 1 and 2 , we obtain the complex $\left.Q_{P_{n} \times\left\langle\sigma_{n}\right.}\right\rangle$ pictured in figure 3 . The complex is homeomorphic to the fletching of a dart, three half disks, all identified along a common line in their boundary. This complex is clearly contractible.

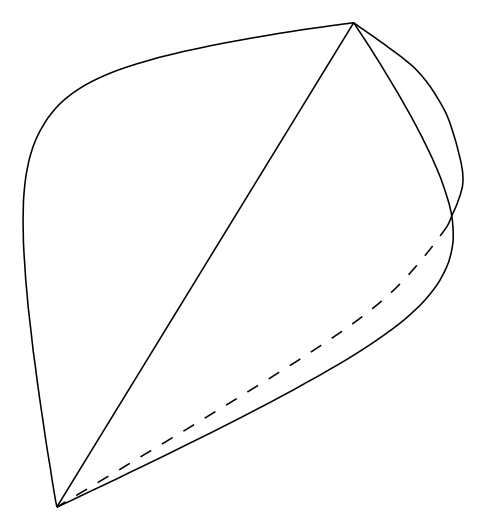

Figure 3. The complete complex $Q_{P_{n} \times\left\langle\sigma_{n}\right\rangle}$

\section{References}

[1] A. Adem and R. J. Milgram, Cohomology of Finite Groups, Springer-Verlag, 1994.

[2] K. Brown, Cohomology of Groups, Springer-Verlag, 1982.

[3] Y. Q. Chen, Farrell cohomology of automorphism groups of free groups of finite rank, Ohio State University Ph.D. dissertation, Columbus, Ohio 1998.

[4] D. J. Collins, Cohomological dimension and symmetric automorphisms of a free group, Comment. Math. Helv. 64 (1989), 44-61.

[5] D. J. Collins, Palindromic automorphisms of free groups, Combinatorial and geometric group theory (Edinburgh, 1993), London Math. Soc. Lecture Note Ser., 204 Cambridge University Press Cambridge (1995), 63-72.

[6] M. Culler, Finite groups of outer automorphisms of a free group, Contemporary Math. 33 (1984), 197-207.

[7] M. Culler and K. Vogtmann, Moduli of graphs and automorphisms of free groups, Invent. Math. 84 (1986), 91-119.

[8] H. M. Farkas and I. Kra, Riemann surfaces, second edition, Springer-Verlag, New York 1992.

[9] F. Thomas Farrell, An extension of Tate cohomology to a class of infinite groups, J. Pure Appl. Algebra 10 (1977), 153-161.

[10] N. D. Gilbert, Presentations of the automorphism group of a free product, Proc. London Math. Soc. (3) 54 (1987), 115-140.

[11] H. H. Glover and G. Mislin, On the $p$-primary cohomology of $\operatorname{Out}\left(F_{n}\right)$ in the $p$ - rank one case, to appear in J. Pure Appl. Algebra 153 (2000), 145-163.

[12] D. Gries, On the cohomology of the hyperelliptic mapping class group, Ohio State University Ph.D. dissertation, Columbus, Ohio 2000.

[13] A. Hatcher, Homological stability for automorphism groups of free groups, Comment. Math. Helv. 70 (1995), 39-62. 
[14] A. Hatcher and K. Vogtmann, Cerf theory for graphs, J. London Math. Soc. (2) 58 (1998), 633-655.

[15] A. Hatcher and K. Vogtmann, Rational homology of Aut $\left(F_{n}\right)$, Math. Res. Lett. 5 (1998), 759-780.

[16] C. A. Jensen, Cohomology of $A u t\left(F_{n}\right)$ in the $p$-rank two case, to appear in J. Pure Appl. Algebra.

[17] C. A. Jensen, Contractibility of fixed point sets of auter space, preprint.

[18] C. A. Jensen, Cohomology of Aut $\left(F_{n}\right)$, Cornell University Ph.D. dissertation, Ithaca, New York 1998.

[19] P. Kropholler and G. Mislin, Groups acting on finite-dimensional spaces with finite stabilizers, Comment. Math. Helv. 73 (1998), 122-136.

[20] S. Krstic, Actions of Finite Groups on Graphs and Related Automorphisms of Free Groups, J. Algebra 124 (1989), 119-138.

[21] S. Krstic and K. Vogtmann, Equivariant outer space and automorphisms of free-by-finite groups, Comment. Math. Helv. 68 (1993), 216-262.

[22] B. Zimmerman, Über Homöomorphismen $n$-dimensionaler Henkelkörper und endliche Erweiterungen von Schottky-Gruppen, Comment. Math. Helv. 56 (1981), 424-486.

Henry H. Glover and Craig A. Jensen

Department of Mathematics

The Ohio State University

Columbus, OH 43210

USA

e-mail: glover@math.ohio-state.edu jensen@math.ohio-state.edu

(Received: January 17, 2000) 